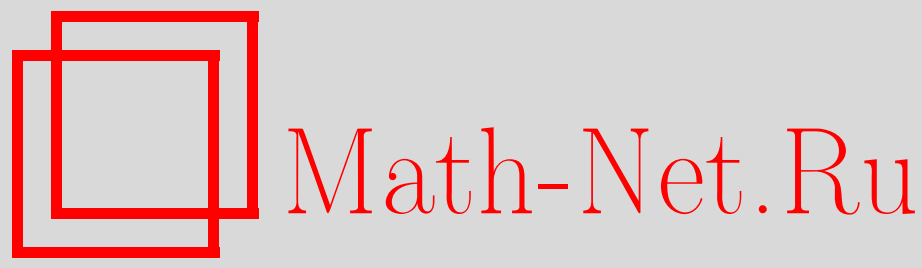

Д. В. Быков, Квазилинейная формулировка $\sigma$-модели пространства флагов, ТМФ, 2017, том 193, номер 3, 381400

DOI: https://doi.org/10.4213/tmf9441

Использование Общероссийского математического портала Math-Net.Ru подразумевает, что вы прочитали и согласны с пользовательским соглашением http://www . mathnet.ru/rus/agreement

Параметры загрузки:

IP : 54.157 .27 .8

26 апреля 2023 г., 05:44:59

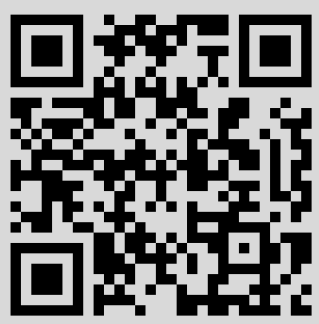




\title{
ФИЗИКА
}

Том 193, № 3

декабрь, 2017

(C) 2017 г.

\author{
Д. В. Быков*
}

\section{КВАЗИЛИНЕЙНАЯ ФОРМУЛИРОВКА $\sigma$-МОДЕЛИ ПРОСТРАНСТВА ФЛАГОВ}

\begin{abstract}
$\sigma$-Модели пространства флагов, обладающие представлением нулевой кривизны, формулируются в виде теории линейных "полей материи", взаимодействующих со вспомогательными калибровочными полями.
\end{abstract}

Ключевые слова: сигма-модели, интегрируемые модели, пространство флагов, кэлеров фактор.

DOI: https://doi.org/10.4213/tmf9441

\section{1. ИССЛЕДУЕМЫЕ МОДЕЛИ}

Мы рассматриваем некоторые двумерные $\sigma$-модели с однородными комплексными таргет-пространствами $\mathcal{M}=\frac{G}{H}$. Обозначим через $\mathbb{G}$ метрику Киллинга на $\mathcal{M}$, а через II - (интегрируемую $G$-инвариантную) комплексную структуру. Будем считать, что метрика эрмитова относительно данной комплексной структуры. Для таких таргет-пространств в работе [1] (как обобщение примеров из работ [2], [3]) были сформулированы $\sigma$-модели с действием

$$
\mathcal{S}[\mathbb{G}, \mathbb{I}]:=\int_{\Sigma} d^{2} z\|\partial X\|_{\mathbb{G}}^{2}+\int_{\Sigma} X^{*} \omega,
$$

где $\omega$ - кэлерова форма, отвечающая паре $(\mathbb{G}, \mathbb{I})$ и определенная формулой

$$
\omega=\mathbb{G} \circ \mathbb{I} .
$$

Особенность данных моделей состоит в том, что их уравнения движения допускают представление нулевой кривизны. Существование такого представления, как правило, является характерной чертой интегрируемых моделей. Дело в том, что для моделей (1) канонический нётеров ток $K$, связанный с группой $G$ глобальной

Исследование выполнено за счет гранта Российского научного фонда (проект № 14-50-00005.

* Математический институт им. В. А. Стеклова Российской академии наук, Москва, Россия. E-mail: dbykov@mi.ras.ru 
симметрии, не только сохраняется (условие сохранения эквивалентно уравнениям движения модели), но также является плоским: $d K-K \wedge K=0$. Это позволяет построить однопараметрическое семейство $\mathcal{A}_{u}, u \in \mathbb{C}$, плоских связностей по классической формуле Полмайера [4]:

$$
A_{u}=1-\frac{u}{2} K_{z} d z+\frac{1-u^{-1}}{2} K_{\bar{z}} d \bar{z} .
$$

Рассматриваемые модели являются каноническим обобщением на случай несимметрических таргет-пространств моделей с эрмитовыми симметрическими таргетпространствами, таких как хорошо известная $\mathbb{C P}^{N-1}$-модель [5]. В настоящей статье нас интересуют в первую очередь пространства флагов - весьма богатый класс комплексных однородных многообразий. Мы рассматриваем группу $G=S U(N)$; соответствующие пространства флагов суть

$$
\mathcal{F}\left(n_{1}, \ldots, n_{m}\right):=\frac{S U(N)}{S\left(U\left(n_{1}\right) \times \cdots \times U\left(n_{m}\right)\right)}, \quad \sum_{i=1}^{m} n_{i}=N .
$$

Многообразие $\mathcal{F}\left(n_{1}, \ldots, n_{m}\right)$ можно рассматривать как (ко)присоединенную орбиту (присоединенное и коприсоединенное представления эквивалентны, если существует невырожденная метрика Киллинга, что выполняется для алгебры $\left.\mathfrak{s u}_{N}\right)$. Это означает, что для моделирования многообразия $\mathcal{F}\left(n_{1}, \ldots, n_{m}\right)$ можно взять элемент $z \in \mathfrak{s u}_{N}$ и рассмотреть его орбиту относительно действия группы:

$$
\operatorname{Orb}(z)=\left\{g z g^{-1}, g \in S U(N)\right\} .
$$

Элемент $z$ нужно выбрать таким образом, чтобы его стабилизатором была подгруппа $H=S\left(U\left(n_{1}\right) \times \cdots \times U\left(n_{m}\right)\right)$. Можно считать, что $z$ лежит в подалгебре Картана, так как, очевидно, каждая орбита $\operatorname{Orb}(z)$ пересекает ее. Следовательно, возьмем в качестве $z$ диагональную матрицу,

$$
\begin{gathered}
z=\operatorname{Diag}(\underbrace{\lambda_{1}, \ldots, \lambda_{1}}_{n_{1}}, \ldots, \underbrace{\lambda_{m}, \ldots, \lambda_{m}}_{n_{m}}), \\
\operatorname{Tr}(z)=\sum_{i=1}^{m} n_{i} \lambda_{i}=0, \quad \lambda_{i} \neq \lambda_{j} \quad \text { для } i \neq j .
\end{gathered}
$$

Для полноты изложения опишем кэлерову структуру на $\operatorname{Orb}(z)=\mathcal{F}\left(n_{1}, \ldots, n_{m}\right)$. В дальнейшем мы сравним ее со структурами, характерными для метрики Киллинга $\mathbb{G}$ на $\mathcal{F}\left(n_{1}, \ldots, n_{m}\right)$, которая в общем случае не является кэлеровой, но это как раз та метрика, которая входит в действие (1). Введя стандартный картановский ток $J:=-g^{-1} d g$, определим симплектическую форму на $\operatorname{Orb}(z)$ следующим образом: $\Omega=\operatorname{tr}(z J \wedge J)$. Эта форма замкнута, что вытекает из уравнения Маурера-Картана $d J-J \wedge J=0$. Чтобы показать, что она является хорошо определенной два-формой на $\frac{G}{H}$, т. е. что она является калибровочно-инвариантной относительно преобразований $J \rightarrow h^{-1} J h-h^{-1} d h$, введем следующее стандартное разложение алгебры Ли:

$$
\mathfrak{g}=\mathfrak{h} \oplus \mathfrak{m}, \quad \mathfrak{m} \perp_{\mathbb{G}} \mathfrak{h} ; \quad[\mathfrak{h}, \mathfrak{h}] \subset \mathfrak{h}, \quad[\mathfrak{h}, \mathfrak{m}] \subset \mathfrak{m} .
$$

Используя соответствующее разложение $J=J_{\mathfrak{h}}+J_{\mathfrak{m}}$ для тока, имеем

$$
\Omega=\operatorname{tr}\left(z J_{\mathfrak{m}} \wedge J_{\mathfrak{m}}\right) .
$$




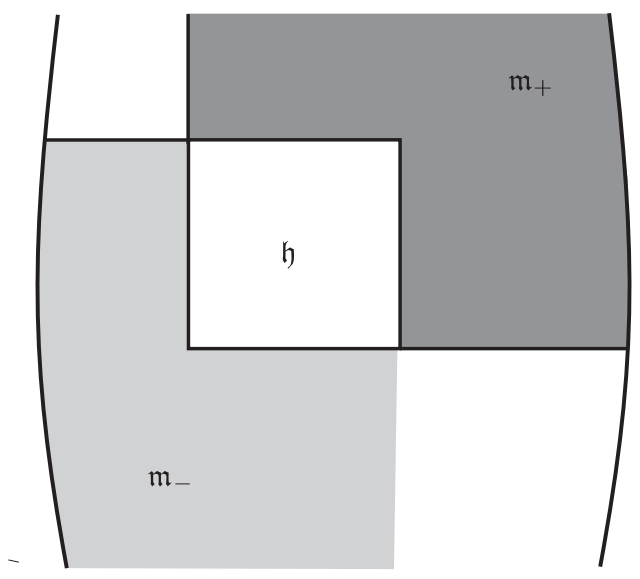

Рис. 1. Разложение (8) алгебры Ли.

При калибровочных преобразованиях компонента $J_{\mathfrak{m}}$ преобразуется однородным образом, $J_{\mathfrak{m}} \rightarrow h^{-1} J_{\mathfrak{m}} h$, и, кроме того, $[z, h]=0$ для $h \in H$, поэтому форма $\Omega$ инвариантна.

Чтобы убедиться, что $\Omega$ - кэлерова форма, необходимо показать, что она является эрмитовой относительно выбранной комплексной структуры $\mathbb{I}$ на $\mathcal{F}\left(n_{1}, \ldots, n_{m}\right)$. Чтобы определить действие данной комплексной структуры, введем в первую очередь комплексную структуру в пространтве $\mathfrak{m}$, обозначив ее при этом той же буквой II. В таком случае комплексификация алгебры Ли $\mathfrak{g}$ допускает разложение

$$
\mathfrak{g}_{\mathbb{C}}=\mathfrak{h} \oplus \mathfrak{m}_{+} \oplus \mathfrak{m}_{-},
$$

где $\mathfrak{m}_{ \pm}$- собственные пространства комплексной структуры: $\mathbb{I} \circ \mathfrak{m}_{ \pm}= \pm i \mathfrak{m}_{ \pm}$.

Разложим теперь стандартный картановский ток $J$ в соответствии с разложением (8) алгебры Ли:

$$
J=J_{\mathfrak{h}}+\underbrace{J_{+}+J_{-}}_{=J_{\mathfrak{m}}} .
$$

Определим комплексную структуру $\mathbb{I}$ на $\mathcal{F}\left(n_{1}, \ldots, n_{m}\right)$ через ее действие на одинформы:

$$
\mathbb{I} \circ J_{ \pm}= \pm i J_{ \pm}
$$

Можно удостовериться, что интегрируемость комплексной структуры эквивалентна условию, что $\mathfrak{m}_{ \pm}-$подалгебры в $\mathfrak{g}_{\mathbb{C}}$ (доказательство можно найти, например, в [1]):

$$
\left[\mathfrak{m}_{ \pm}, \mathfrak{m}_{ \pm}\right] \subset \mathfrak{m}_{ \pm}
$$

С точностью до действия группы Вейля (т. е. с точностью до перестановки), можно считать, что $\mathfrak{m}_{+}-$пространство блочных верхнетреугольных матриц (на рис. 1 изображен пример, соответствующий $m=3)$. В силу условия (11)

$$
\operatorname{tr}\left(z J_{+} \wedge J_{+}\right)=\operatorname{tr}\left(z J_{-} \wedge J_{-}\right)=0
$$


(так как $z \perp \mathfrak{m}_{ \pm}$), поэтому форма $\Omega$ эрмитова и, следовательно, кэлерова.

Одно из следствий данного рассуждения состоит в том, что кэлеровы формы на пространстве флагов $(4)$ образуют $(m-1)$-параметрическое семейство (параметрами служат $\lambda_{i}$, см. (5)). В разделе 3 мы увидим, как данные параметры появляются в рамках другого подхода - теории кэлерова фактора. А пока прервемся, чтобы ответить на следующий вопрос: в каком случае метрика Киллинга $\mathbb{G}$, входящая в действие (1), является кэлеровой? Оказывается, что это имеет место лишь в случае симметрических пространств.

ПрЕДЛОЖЕНИЕ 1. Метрика Киллинга на $\mathcal{F}\left(n_{1}, \ldots, n_{m}\right)$ является кэлеровой тогда и только тогда, когда $\mathcal{F}\left(n_{1}, \ldots, n_{m}\right)$ - симметрическое пространство, т.е. $m=2, u \mathcal{F}\left(n_{1}, N-n_{1}\right)$ - грассманиан.

ДоказАтельство. Согласно определению (10) компоненты $J_{ \pm}$тока $J$ (см. разложение (9)) представляют собой голоморфные/антиголоморфные один-формы, т. е.

$$
J_{+} \in\left(T^{*} \mathcal{F}\right)^{(1,0)}, \quad J_{-} \in\left(T^{*} \mathcal{F}\right)^{(0,1)} .
$$

Следовательно, метрика Киллинга на $\mathcal{F}$, которая имеет вид $d s^{2}=-\operatorname{Tr}\left(J_{+} J_{-}\right)$, эрмитова. Кэлерова форма строится стандартным способом:

$$
\omega=i \operatorname{Tr}\left(J_{+} \wedge J_{-}\right)
$$

При вычислении ее внешней производной воспользуемся условием $d J-J \wedge J=0$, согласно которому ток является плоским:

$$
d \omega=i \operatorname{Tr}\left(d J_{+} \wedge J_{-}\right)-i \operatorname{Tr}\left(J_{+} \wedge d J_{-}\right)=i \operatorname{Tr}\left((J \wedge J)_{\mathfrak{m}_{+}} \wedge J_{-}\right)-i \operatorname{Tr}\left(J_{+} \wedge(J \wedge J)_{\mathfrak{m}_{-}}\right) .
$$

Отметим, что $\mathfrak{m}_{+} \perp \mathfrak{h}$ и $\mathfrak{m}_{+} \perp \mathfrak{m}_{+}$(т. е. пространство $\mathfrak{m}_{+}$изотропно, так как метрика эрмитова). Поэтому $\operatorname{Tr}\left(J_{+} \wedge(J \wedge J)_{\mathfrak{m}_{-}}\right)=\operatorname{Tr}\left(J_{+} \wedge J \wedge J\right)$ и аналогично для первого слагаемого в (13). В результате получаем для $d \omega$ следующее выражение:

$$
d \omega=i \operatorname{Tr}\left(J \wedge J \wedge J_{-}\right)-i \operatorname{Tr}\left(J_{+} \wedge J \wedge J\right) .
$$

Подставляя разложение $(9)$ и используя вновь условия ортогональности $\mathfrak{h} \perp \mathfrak{m}_{ \pm}$, $\mathfrak{m}_{ \pm} \perp \mathfrak{m}_{ \pm}$, получаем

$$
d \omega=i\left(\operatorname{Tr}\left(J_{+} \wedge J_{-} \wedge J_{-}\right)-\operatorname{Tr}\left(J_{-} \wedge J_{+} \wedge J_{+}\right)\right) .
$$

Три-формы в правой части этого равенства имеют относительно разложения Ходжа тип $(1,2)$ и $(2,1)$ (они комплексно сопряжены друг другу). Следовательно, $d \omega=0$ тогда и только тогда, когда $\operatorname{Tr}\left(J_{+} \wedge J_{-} \wedge J_{-}\right)=0$. Можно показать, что последнее условие эквивалентно следующему:

$$
\operatorname{Tr}\left(\alpha_{+}\left[\beta_{-}, \gamma_{-}\right]\right)=0 \quad \text { для } \quad \alpha_{+} \in \mathfrak{m}_{+} \quad \text { и } \quad \beta_{-}, \gamma_{-} \in \mathfrak{m}_{-} .
$$

Ввиду невырожденности метрики Киллинга это может иметь место при произвольных $\alpha_{+}, \beta_{-}, \gamma_{-}$, только если $\left[\mathfrak{m}_{-}, \mathfrak{m}_{-}\right]=0$. Из рис. 1 легко видеть, что данное условие выполняется лишь в случае $m=2$. Можно также напрямую показать, что свойство $\left[\mathfrak{m}_{-}, \mathfrak{m}_{-}\right]=0$ является характеристическим для эрмитовых симметричных 
пространств. Действительно, пусть $\alpha_{+} \in \mathfrak{m}_{+}$, а $\beta_{-}, \gamma_{-} \in \mathfrak{m}_{-}$, как и выше. Ранее мы наложили условие $\left[\mathfrak{m}_{-}, \mathfrak{m}_{-}\right] \subset \mathfrak{m}_{-}$, что вкупе с условием $[\mathfrak{m}, \mathfrak{m}] \subset \mathfrak{h}$ для симметрического пространства дает $\left[\mathfrak{m}_{-}, \mathfrak{m}_{-}\right]=0$.

В обратную сторону, пусть $\left[\mathfrak{m}_{-}, \mathfrak{m}_{-}\right]=0$, тогда

$$
\operatorname{Tr}\left(\left[\alpha_{+}, \beta_{-}\right] \gamma_{-}\right)=\operatorname{Tr}\left(\alpha_{+}\left[\beta_{-}, \gamma_{-}\right]\right)=0,
$$

т. е. $\left[\mathfrak{m}_{+}, \mathfrak{m}_{-}\right] \perp \mathfrak{m}_{-}$. Аналогичным образом выводим, что $\left[\mathfrak{m}_{+}, \mathfrak{m}_{-}\right] \perp \mathfrak{m}_{+}$. Отсюда следует, что $\left[\mathfrak{m}_{+}, \mathfrak{m}_{-}\right] \subset \mathfrak{h}$, поэтому и $[\mathfrak{m}, \mathfrak{m}] \subset \mathfrak{h}$, тем самым предложение доказано.

Из предложения 1 следует, что когда таргет-пространство есть грассманиан, т. е. $m=2$, второе слагаемое в действии (1) на самом деле является топологическим и не вносит вклад в уравнения движения. В этом случае мы возвращаемся к хорошо известной теории интегрируемых $\sigma$-моделей с симметрическими таргет-пространствами [6], [7]. С другой стороны, когда $m>2$, модели (1) отличаются от стандартных ненулевым (и полностью фиксированным) $B$-полем. Простейший пример, который мы изучим детально в п. 2.2 , отвечает пространству флагов $\mathcal{F}_{3}:=\frac{S U(3)}{S\left(U(1)^{3}\right)}$, т. е. случаю $m=3$.

\section{2. КВАЗИЛИНЕЙНАЯ ФОРМУЛИРОВКА $\sigma$-МОДЕЛЕЙ}

Основная задача настоящей работы - построение квазилинейного представления для $\sigma$-моделей (1) в случае, когда таргет-пространство есть пространство флагов (4). Под квазилинейным представлением мы понимаем так называемую "gauged linear $\sigma$-model" (GLSM), т. е. теорию нескольких свободных полей, подчиняющихся квадратичным условиям связи и взаимодействующих со вспомогательными калибровочными полями ${ }^{1)}$.

Ключевой шаг при построении квазилинейного представления $\sigma$-моделей с симметрическими кэлеровыми таргет-пространствами (например, когда таргет-пространство есть грассманиан) состоит в представлении данных многообразий в виде кэлерова фактора плоского пространства $\mathbb{C}^{N}$ для некоторого $N$. Подобные представления получаются наиболее естественным способом в рамках суперсимметричной $\mathcal{N}=(2,2)$ формулировки, которая автоматически сохраняет условие кэлеровости (в п. 3.2 работы [8] подробно разобран случай $\mathbb{C P}^{N-1}$-модели). В предыдущем разделе мы уже, однако, подчеркивали, что метрика Киллинга, входящая в действие (1), вообще говоря, не является кэлеровой, в связи с чем подход, основанный на суперсимметрии, не является для наших моделей наиболее естественным. Поэтому мы остаемся в рамках бозонных моделей и демонстрируем, каким образом подходящая модификация кэлерова фактора все же приводит нас к требуемой квазилинейной формулировке моделей (1).

2.1. $\mathbb{C P}^{N-1}$-модель как прототип. Простейший грассманиан - это комплексное проективное пространство $\mathbb{C P}^{N-1}$. Хорошо известно, что его можно представить в виде кэлерова фактора:

$$
\mathbb{C} \mathbb{P}^{N-1}=\mathbb{C}^{N} / / U(1)
$$

Группа $U(1)$ действует на пространстве $\mathbb{C}^{N}$ умножением всех координат на общую фазу: $z_{k} \rightarrow e^{i \alpha} z_{k}$, где $\left\{z_{k}, k=1, \ldots, N\right\}$ - комплексные координаты на $\mathbb{C}^{N}$. Кэлеров

1)В отечественной литературе соответствующий термин, по-видимому, отсутствует. 
фактор обычно строится следующим образом: прежде всего, рассмотрим кэлерову форму на $\mathbb{C}^{N}$,

$$
\omega:=i \sum_{j=1}^{N} d z_{j} \wedge d \bar{z}_{j}
$$

Пусть $v$ - векторное поле, отвечающее $U(1)$-действию, т. е.

$$
v=\operatorname{Re}\left(i \sum_{k=1}^{N} z_{k} \frac{\partial}{\partial z_{k}}\right) .
$$

Отображение момента $\mu$ определяется стандартной формулой

$$
d \mu=i_{v} \omega
$$

где $i_{v}$ - свертка с векторным полем $v$. Используя приведенные определения, получаем следующую формулу для $\mu$ (с точностью до аддитивной константы):

$$
\mu=\sum_{k=1}^{N}\left|z_{k}\right|^{2} \text {. }
$$

Чтобы построить фактор, ограничим кэлерову форму $\omega$ на множество уровня

$$
\mu=\mu_{0}>0
$$

В данном случае это сфера $S^{2 N-1}$. Ограниченная форма является вырожденной: ее ядро $\operatorname{Ker}\left(\left.\omega\right|_{\mu=\mu_{0}}\right)$ одномерно - его составляют векторы, пропорциональные $v$. Это легко видеть из (19): для любого вектора $w$, касательного к $\mu=\mu_{0}$, имеет место равенство $i_{w} d \mu=0$, поэтому $\omega(v, w)=0$ для всех $w \in T\left(\mu=\mu_{0}\right)$. Чтобы получить невырожденную форму, необходимо рассмотреть фактор-многообразие $\left\{\mu=\mu_{0}\right\} / U(1)$, т. е. отождествить все точки вдоль каждой $U(1)$-орбиты. Для нашего примера имеем $S^{2 N-1} / U(1)=\mathbb{C P}^{N-1}$, а фактор - это расслоение Хопфа.

Представление (17) - не просто схоластическое упражнение, оно является весьма полезным для анализа соответствующей $\sigma$-модели. Используя его, можно очень просто записать лагранжиан модели:

$$
\mathcal{L}=\sum_{k=1}^{N} \overline{\mathscr{D}_{\alpha} Z_{k}} \mathscr{D}_{\alpha} Z_{k}+\lambda\left(\sum_{k=1}^{N}\left|Z_{k}\right|^{2}-\mu_{0}\right) .
$$

Здесь $\lambda$ - множитель Лагранжа, обеспечивающий ограничение полей на множество уровня $\mu=\mu_{0}$, а $\mathscr{D}$ - ковариантная производная группы $U(1)$,

$$
\mathscr{D}_{\alpha}=\partial_{\alpha}-i \mathcal{A}_{\alpha}
$$

Из (22) можно получить обычное "нелинейное" представление для $\sigma$-модели с таргет-пространством $\mathbb{C P}^{N-1}$, если исключить при помощи уравнений движения вспомогательное калибровочное поле $\mathcal{A}_{\alpha}$. Преимущество представления (22), однако, состоит в том, что лагранжиан квадратичен по "полям материи" $Z_{k}$. В частности, в континуальном интеграле оно позволяет с легкостью проинтегрировать по полям материи. Квазилинейное представление $(22)$ для $\mathbb{C P}^{N-1}$-модели впервые появилось в работах [9], [10]. 
2.2. Многообразие полных флагов в $\mathbb{C}^{3}$. Заметим, что $\frac{S U(2)}{S(U(1) \times U(1))}=S^{2}$ есть сфера, т. е. симметрическое пространство, следовательно, для группы $G=S U(2)$ не существует несимметрических пространств флагов. Поэтому перейдем к группе $G=S U(3)$ и нашему основному примеру - простейшему несимметрическому пространству флагов

$$
\mathcal{F}_{3}=\frac{S U(3)}{S\left(U(1)^{3}\right)}
$$

Его можно рассматривать как многообразие линейных вложенных пространств (проходящих через начало координат)

$$
\mathcal{F}_{3}=\left\{L_{1} \subset L_{2} \subset \mathbb{C}^{3}\right\}, \quad L_{1} \simeq \mathbb{C}, \quad L_{2} \simeq \mathbb{C}^{2}
$$

Одна из параметризаций данного многообразия, которая лежит в основе GLSM-формулировки, заключается в следующем. Пусть $l, p \in \mathbb{C}^{3}$ - два ортонормированных вектора:

$$
\sum_{i=1}^{3} \bar{l}^{i} l^{i}=\sum_{i=1}^{3} \bar{p}^{i} p^{i}=1, \quad \sum_{i=1}^{3} \bar{l}^{i} p^{i}=0 .
$$

Эти векторы задают плоскость

$$
L_{2}=\operatorname{Span}(l, p) \simeq \mathbb{C}^{2} \subset \mathbb{C}^{3}
$$

Прямая $L_{1} \subset L_{2}$ определяется вектором

$$
L_{1}=\operatorname{Span}\left(\bar{u}_{1} l+\bar{u}_{2} p\right) \subset L_{2} \subset \mathbb{C}^{3},
$$

где $\left(u_{1}, u_{2}\right)$ - фиксированный ненулевой два-вектор, нормированный на единицу:

$$
\bar{u} \circ u=1
$$

(кружок 。 обозначает свертку индекса в $\left.\mathbb{C}^{2}\right)$. Ясно, что векторы $\left(u_{1}, u_{2}\right) \in \mathbb{C}^{2}$, $l \in \mathbb{C}^{3}, p \in \mathbb{C}^{3}$ однозначно определяют флаг $L_{1} \subset L_{2} \subset \mathbb{C}^{3}$, однако это соотношение не взаимно однозначно. Действительно, другой набор векторов

$$
\left(\begin{array}{c}
\tilde{u}_{1} \\
\tilde{u}_{2}
\end{array}\right)=e^{i \beta} g \circ\left(\begin{array}{c}
u_{1} \\
u_{2}
\end{array}\right), \quad\left(\begin{array}{c}
\tilde{l} \\
\tilde{p}
\end{array}\right)=g \circ\left(\begin{array}{l}
l \\
p
\end{array}\right), \quad \text { где } \quad g \in U(2), e^{i \beta} \in U(1),
$$

определяет тот же флаг и удовлетворяет тем же условиям нормировки (26), (29). Поэтому имеется калибровочная группа

$$
\mathcal{G}=U(1) \times U(2)
$$

действующая на “поля материи”, которые образуют векторное пространство

$$
V=\left(\mathbb{C}^{2}\right)_{u} \oplus\left(\mathbb{C}^{2} \otimes \mathbb{C}^{3}\right)_{l, p}
$$

и удовлетворяют дополнительным условиям нормировки (26), (29) - аналогам условия $\mu=\mu_{0}$ на отображение момента из формулы (21). Параметризуем по-новому 


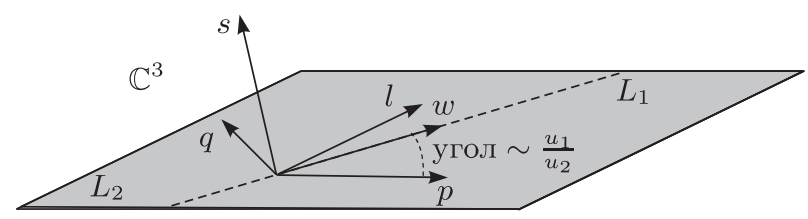

Рис. 2. Параметризация пространства флагов, введенная в (26)-(28), (34)-(37).

элементы пространства $\mathbb{C}^{2} \otimes \mathbb{C}^{3}$ : выше они были сгруппированы в два $S U(3)$-триплета $l, p$, а теперь мы сгруппируем их в три $U(2)$-дублета

$$
v^{i}=\left(\begin{array}{c}
l^{i} \\
p^{i}
\end{array}\right), \quad i=1,2,3 .
$$

Условие нормировки (26) принимает вид

$$
\sum_{i=1}^{3} \bar{v}^{i} \otimes v^{i}=\mathbb{1}_{2}
$$

Соответствующий квазилинейный лагранжиан - аналог (22) - определим следующим образом (опустим для простоты часть лагранжиана, содержащую множители Лагранжа, обеспечивающие выполнение условий нормировки (29), (32)):

$$
\mathcal{L}=\frac{1}{\kappa} \overline{\mathscr{D}_{z} u} \circ \mathscr{D}_{z} u+\sum_{k=1}^{3} \overline{\mathscr{D}_{z} v^{k}} \circ \mathscr{D}_{z} v^{k}
$$

Здесь $\kappa$ - относительный множитель между двумя членами в лагранжиане - константа связи, а ковариантные производные действуют в соответствии с зарядами полей, т. е.

$$
\mathscr{D}_{z} u=\partial_{z} u-i \mathcal{A}_{z} u-i \mathcal{B}_{z} u, \quad \mathscr{D}_{z} v^{k}=\partial_{z} v^{k}-i \mathcal{B}_{z} v^{k}
$$

В этих формулах $i \mathcal{B} \in \mathfrak{u}(2)$ и $i \mathcal{A} \in \mathfrak{u}_{1}$ - соответствующие калибровочные поля группы $\mathcal{G}$. Поля $\mathcal{A}$ и $\mathcal{B}$ эрмитовы. Потребуем также, чтобы действие было неотрицательно, следовательно, $\kappa \geqslant 0$. Возможно, на первый взгляд покажется удивительным тот факт, что в данной модели нас будет прежде всего интересовать предел $\kappa \rightarrow 0$. С точки зрения лагранжиана (33) этот предел кажется несколько сингулярным, однако сейчас мы убедимся, что на самом деле наша модель хорошо определена в данном пределе.

Стратегия состоит в том, чтобы, как и в случае $\mathbb{C P}^{N-1}$-модели, выразить вспомогательные калибровочные поля через поля материи. Для этого введем три вектора $w, q, s \in \mathbb{C}^{3}$, задающие флаг $L_{1} \subset L_{2} \subset \mathbb{C}^{3}$. Начнем с того, что прямая $L_{1}$ порождена вектором

$$
w^{k}:=\bar{u} \circ v^{k}
$$

Из условия нормировки $(32)$ следует, что $(3 \times 3)$-матрица

$$
P^{m n}:=v^{m} \circ \bar{v}^{n}
$$


является проектором ранга два, т. е. $P \cdot P=P$, rank $P=2$. Кроме того, из определения (34) вектора $w$ и из (32) можно видеть, что $P w=w$. Следовательно, проектор $P$ можно записать в виде

$$
P=w \otimes \bar{w}+q \otimes \bar{q},
$$

где $q \in \mathbb{C}^{3}$ - некоторый вектор, ортогональный $w$ и нормированный на единицу. Мы рассматриваем формулу (36) как определение вектора $q$. Введем теперь третий вектор $s$, ортогональный как $w$, так и $q$, при помощи соотношения полноты в $\mathbb{C}^{3}$ (см. рис. 2):

$$
\mathbb{1}_{3}=P+s \otimes \bar{s}=w \otimes \bar{w}+q \otimes \bar{q}+s \otimes \bar{s} .
$$

Резюмируем результат исключения вспомогательных калибровочных полей из лагранжиана (33) (детали приведены в приложении А). Во-первых, в пределе $\kappa \rightarrow 0$ ковариантная производная $\mathscr{D}_{z} u=O(\kappa)$, а для $\mathscr{D}_{z} v^{k}$ в том же пределе имеем выражение

$$
\mathscr{D}_{z} v^{k}=-w^{k} \sum_{m=1}^{3}\left(v^{m} \partial_{z} \bar{w}^{m}+u \bar{w}^{m} \partial_{z} w^{m}\right)-s^{k} \sum_{m=1}^{3} v^{m} \partial_{z} \bar{s}^{m} .
$$

Теперь можно вычислить значение лагранжиана (33) после подстановки калибровочных полей и в пределе $\kappa \rightarrow 0$ :

$$
\mathcal{L}=\left|\sum_{m=1}^{3} \bar{q}^{m} \partial_{\bar{z}} s^{m}\right|^{2}+\left|\sum_{m=1}^{3} \bar{w}^{m} \partial_{\bar{z}} s^{m}\right|^{2}+\left|\sum_{m=1}^{3} \bar{q}^{m} \partial_{\bar{z}} w^{m}\right|^{2} .
$$

Мы получили лагранжиан, совпадающий с исследованным ранее в нашей статье [2].

Описанные вычисления были проведены в пределе $\kappa \rightarrow 0$. При исключении калибровочных полей можно также считать $\kappa \neq 0$, при этом в результате мы снова получим $\sigma$-модель пространства флагов, но с метрикой, зависящей от $\kappa$ и имеющей гладкий предел $\kappa \rightarrow 0$. Подробные вычисления приведены в приложении Б.

2.3. Нётеров ток. Лагранжиан (33) обладает глобальной $U(3)$-симметрией, которая действует по правилу $v^{k} \rightarrow \sum_{m=1}^{3} \hat{g}_{k m} v^{m}, \hat{g} \in U(3)$. Компоненты соответствующего нётерова тока таковы:

$$
K^{m \bar{n}}=\overline{\mathscr{D}_{z} v^{n}} \circ v^{m} d \bar{z}-\bar{v}^{n} \circ \mathscr{D}_{z} v^{m} d z, \quad m, n=1,2,3
$$

(напомним, что кружок обозначает свертку по $U(2)$-индексу). Из уравнений движения следует, что нётеров ток сохраняется: $d * K^{m \bar{n}}=0$ (здесь $*-$ звездочка Ходжа). Подставляя выражение (38) для ковариантной производной $\mathscr{D}_{z} v^{m}$, получаем

$$
K_{z}^{m \bar{n}}=\bar{q}^{n} w^{m}\left(\sum_{j} q^{j} \partial_{z} \bar{w}^{j}\right)+\bar{w}^{n} s^{m}\left(\sum_{j} w^{j} \partial_{z} \bar{s}^{j}\right)+\bar{q}^{n} s^{m}\left(\sum_{j} q^{j} \partial_{z} \bar{s}^{j}\right) .
$$

Вводя матрицу $g:=(s, w, q)$, можно записать ток в этом базисе:

$$
K_{z}=g\left(\begin{array}{ccc}
0 & \sum_{j} w^{j} \partial_{z} \bar{s}^{j} & \sum_{j} q^{j} \partial_{z} \bar{s}^{j} \\
0 & 0 & \sum_{j} q^{j} \partial_{z} \bar{w}^{j} \\
0 & 0 & 0
\end{array}\right) g^{\dagger}
$$




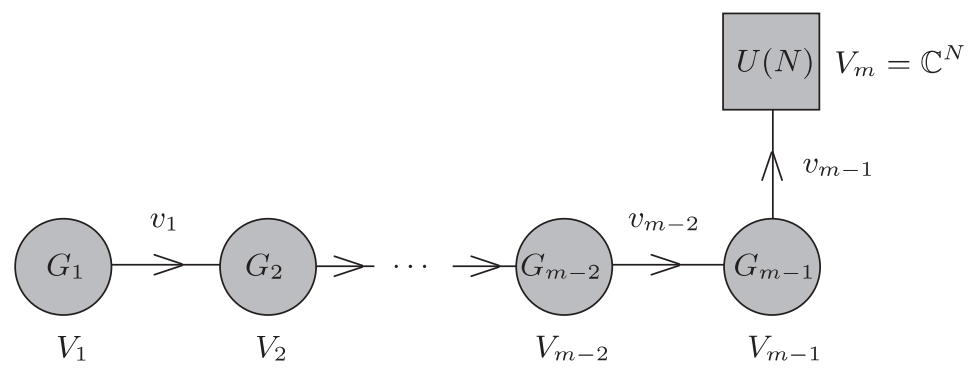

Рис. 3. Колчанная диаграмма, описывающая кэлеров фактор для пространства флагов.

Заметим, что данный ток оказывается бесследовым, так как $U(1)$-часть симметрии $\left(v^{k} \rightarrow e^{i \alpha} v_{k}\right)$ на самом деле включена в калибровочную группу $U(2)$ и поэтому не имеет соответствующего нётерова тока (т. е. настоящей группой глобальных симметрий является $S U(3))$.

В работе [2] показано, что (41) - в точности выражение для нётерова тока, которое получается из лагранжиана (39). Условие его сохранения эквивалентно уравнениям движения, вытекающим из этого лагранжиана. Кроме того, если выполнены уравнения движения, то этот ток также является плоским, т. е. $d K-K \wedge K=0$. Отсюда следует, что связности из однопараметрического семейства $A_{u}, u \in \mathbb{C}$, заданного формулой (3), плоские.

\section{3. ПРОСТРАНСТВА ФЛАГОВ ОБЩЕГО ВИДА: КЭЛЕРОВ ФАКТОР, ЗАДАННЫЙ КОЛЧАННОЙ ДИАГРАММОЙ}

Описанную выше процедуру можно обобщить на случай пространств флагов общего вида $\mathcal{F}\left(n_{1}, \ldots, n_{m}\right)$. При этом необходимо иметь аналог данных $(30),(31)$ : нужно задать пространство $V$ полей материи, а также калибровочную группу $\mathcal{G}$, действующую на эти поля. Для этого можно воспользоваться общей теорией так называемых "представлений колчанов" (quiver representations) [11]. Напомним, что пространство флагов $\mathcal{F}\left(n_{1}, \ldots, n_{m}\right)$ можно рассматривать как многообразие вложенных линейных пространств (проходящих через начало координат)

$$
\mathcal{F}\left(n_{1}, \ldots, n_{m}\right)=\left\{V_{1} \subset V_{2} \subset \cdots \subset V_{m}=\mathbb{C}^{N}\right\}, \quad d_{k}:=\operatorname{dim} V_{k}=\sum_{i=1}^{k} n_{i} .
$$

Позаимствуем из общей теории следующий факт: пространство (43) можно представить в виде кэлерова фактора

$$
\mathcal{F}\left(n_{1}, \ldots, n_{m}\right)=\widehat{V} / / \widehat{\mathcal{G}}, \quad \widehat{V}=\bigoplus_{i=1}^{m-1} \operatorname{Hom}\left(V_{i}, V_{i+1}\right), \quad \widehat{\mathcal{G}}=\bigotimes_{k=1}^{m-1} U\left(V_{k}\right) .
$$

В дальнейшем мы будем взаимозаменяемо использовать обозначения $U\left(V_{k}\right)$ и $U\left(d_{k}\right)$, так как $d_{k}=\operatorname{dim} V_{k}$. Данные (44) можно представить при помощи колчанной диаграммы, изображенной на рис. 3. На данной диаграмме $i$-я вершина отвечает векторному пространству $V_{i}$, в котором действует группа $U\left(V_{i}\right)$, а каждое ребро соответствует полю $v_{i} \in \operatorname{Hom}\left(V_{i}, V_{i+1}\right)$, преобразующемуся по бифундаментальному 
представлению относительно групп, находящихся на концах ребра. То обстоятельство, что последняя вершина отличается от других, призвано подчеркнуть, что отвечающая ей группа $U(N)$ является группой глобальных симметрий, т. е. по ней факторизация не производится (она не включена в группу калибровочной симметрии $\widehat{\mathcal{G}})$.

Зададим на пространстве $\widehat{V}$ кэлерову метрику

$$
d s^{2}=\sum_{k=1}^{m-1} \operatorname{Tr}\left(d v_{k} d v_{k}^{\dagger}\right)
$$

при этом кэлерова форма имеет вид

$$
\Omega=i \sum_{k=1}^{m-1} \operatorname{Tr}\left(d v_{k} \wedge d v_{k}^{\dagger}\right)
$$

Отображение момента $\mu_{k}$ для действия группы $U\left(V_{k}\right)$ имеет вид $\mu_{k}=v_{k}^{\dagger} v_{k}-v_{k-1} v_{k-1}^{\dagger}$, и уравнения кэлерова фактора таковы $\left(v_{0}=0\right)$ :

$$
v_{k}^{\dagger} v_{k}-v_{k-1} v_{k-1}^{\dagger}=\zeta_{k} \mathbb{1}_{V_{k}}, \quad k=1, \ldots, m-1,
$$

где $\zeta_{k} \in \mathbb{R}$ - константы, которые в контексте суперсимметричных калибровочных теорий называются параметрами Файе-Илиополуса. Будем считать, что $\zeta_{k}>0$ для всех $k$. Тогда $\operatorname{Ker}\left(v_{k}\right)=0$. Действительно, умножая (47) на $a$ справа и на $a^{\dagger}$ слева, получаем

$$
\left\|v_{k} \circ a\right\|^{2}=\zeta_{k}\|a\|^{2}+\left\|v_{k-1}^{\dagger} \circ a\right\|^{2}>0 \quad \Longrightarrow \quad v_{k} \circ a \neq 0 \quad(\text { или } \quad a=0) .
$$

Поэтому при любом $k$ матрица $v_{m-1} v_{m-2} \ldots v_{k}$ имеет максимальный ранг $d_{k}$ и заряжена только относительно калибровочной группы $U\left(d_{k}\right)$. Тем самым она определяет подпространство $V_{k} \subset \mathbb{C}^{N}$. Эти подпространства вложены друг в друга, т. е. $V_{k-1} \subset V_{k}$. Следовательно, они задают флаг в $\mathbb{C}^{N}$. Параметры $\zeta_{k}$ связаны с параметрами $\lambda_{i}$ коприсоединенных орбит (см. (5)).

Как уже было неоднократно отмечено выше, в лагранжиан (1) рассматриваемых нами моделей входит не кэлерова метрика, а метрика Киллинга. Поэтому описанную выше конструкцию кэлерова фактора стоит рассматривать лишь как некий намек на правильную геометрическую картину квазилинейного представления. На самом деле основное различие состоит в том, что вместо “условия нормировки" (47) мы будем использовать более простое условие

$$
v_{k}^{\dagger} v_{k}=\mathbb{1}_{V_{k}}, \quad k=1 \ldots m-1
$$

Данное условие в точности совпадает с тем, что было введено нами выше, в формулах (26), (29), (32), для случая пространства флагов $\mathcal{F}_{3}$. Несложно убедиться, что действием группы $U\left(V_{k}\right) \times U\left(V_{k+1}\right)$ можно привести матрицу $v_{k}$ (при $k \leqslant m-2$ ) 
к каноническому виду

$$
v_{k}^{[0]}=\left(\begin{array}{cccc}
1 & 0 & \cdots & 0 \\
0 & 1 & \cdots & 0 \\
\vdots & & \ddots & \vdots \\
0 & 0 & \cdots & 1 \\
0 & 0 & \cdots & 0 \\
\vdots & \vdots & \ddots & \vdots \\
0 & 0 & \cdots & 0
\end{array}\right), \quad k \leqslant m-2
$$

Ясно, что эта матрица удовлетворяет уравнению (48). Несложно определить стабилизатор $H_{k} \subset U\left(V_{k}\right) \times U\left(V_{k+1}\right)$ матрицы $v_{k}^{[0]}: H_{k}=U\left(d_{k}\right) \times U\left(d_{k+1}-d_{k}\right)$ (напомним, что $\left.d_{k}=\operatorname{dim} V_{k}\right)$. Поэтому можно по индукции привести все матрицы $v_{k}$ в порядке убывания индекса $k$ к данной канонической форме: нужно начать с $v_{m-2}$, затем взять $v_{m-3}$ и т. д. В данной "калибровке" вся динамическая информация будет содержаться в $v_{m-1}$. Стабилизатор всего множества матриц $\left\{v_{1}^{[0]}, v_{2}^{[0]}, \ldots, v_{m-2}^{[0]}\right\}$ есть

$$
H=U\left(d_{1}\right) \times U\left(d_{2}-d_{1}\right) \times \cdots \times U\left(N-d_{m-1}\right),
$$

т. е. мы получили в точности стабилизатор, который входит в фактор-представление пространства $\frac{G}{H}=\frac{U(N)}{H}$, как в формуле (4), с точностью до безобидного множителя $U(1)$ в числителе и знаменателе.

По аналогии с (33) запишем лагранжиан

$$
\mathcal{L}=\frac{1}{\kappa} \sum_{k=1}^{m-2} \operatorname{Tr}\left(\left(\mathscr{D}_{z} v_{k}\right)^{\dagger} \circ \mathscr{D}_{z} v_{k}\right)+\operatorname{Tr}\left(\left(\mathscr{D}_{z} v_{m-1}\right)^{\dagger} \circ \mathscr{D}_{z} v_{m-1}\right),
$$

где

$$
\mathscr{D}_{z} v_{k}=\partial_{z} v_{k}+i v_{k}\left(\mathcal{A}_{k}\right)_{z}-i\left(\mathcal{A}_{k+1}\right)_{z} v_{k}
$$

Здесь $\mathcal{A}_{k}$ - эрмитово калибровочное поле для группы $G_{k}=U\left(d_{k}\right)$, и по определению мы также положим $\mathcal{A}_{m}=0$. Прямое исключение калибровочных полей, аналогичное тому, которое было выполнено в п. 2.2, весьма трудоемко, поэтому мы пойдем по другому пути и наложим сразу калибровочное условие (49).

Вариация действия, отвечающего лагранжиану $(51)$, по полю $\left(\mathcal{A}_{k}\right)_{\bar{z}}$ при $k<m-1$ дает уравнение

$$
-v_{k}^{\dagger} \mathscr{D}_{z} v_{k}+\mathscr{D}_{z} v_{k-1} v_{k-1}^{\dagger}=0
$$

следовательно,

$$
-v_{k}^{\dagger}\left(v_{k} \mathcal{A}_{k}-\mathcal{A}_{k+1} v_{k}\right)+\left(v_{k-1} \mathcal{A}_{k-1}-\mathcal{A}_{k} v_{k-1}\right) v_{k-1}^{\dagger}=0,
$$

где $\mathcal{A}_{k} \equiv\left(\mathcal{A}_{k}\right)_{z}$ (опустим временно индекс $z$, чтобы упростить обозначения). Последнее уравнение

$$
-v_{m-1}^{\dagger} \mathscr{D}_{z} v_{m-1}+\frac{1}{\kappa} \mathscr{D}_{z} v_{m-2} v_{m-2}^{\dagger}=0
$$

полученное вариацией по $\left(\mathcal{A}_{m-1}\right)_{\bar{z}}$, можно записать аналогичным образом как

$$
-v_{m-1}^{\dagger}\left(v_{m-1} \mathcal{A}_{m-1}-\widetilde{\mathcal{A}}_{m} v_{m-1}\right)+\frac{1}{\kappa}\left(v_{m-2} \mathcal{A}_{m-2}-\mathcal{A}_{m-1} v_{m-2}\right) v_{m-2}^{\dagger}=0,
$$


но с дополнительным относительным множителем $1 / \kappa$ и с отождествлением

$$
\widetilde{\mathcal{A}}_{m}:=i \partial_{z} v_{m-1} v_{m-1}^{\dagger} .
$$

Покажем сначала, что записанные уравнения можно решить рекуррентно, начиная с $\widetilde{\mathcal{A}}_{m}$ (данное поле уже нам известно), далее вычисляя $\mathcal{A}_{m-1}$ и т. д. Действительно, из (55) получаем

$$
\mathcal{A}_{m-1}\left(1+\frac{v_{m-2} v_{m-2}^{\dagger}}{\kappa}\right)=\frac{1}{\kappa} v_{m-2} \mathcal{A}_{m-2} v_{m-2}^{\dagger}+v_{m-1}^{\dagger} \widetilde{\mathcal{A}}_{m} v_{m-1} .
$$

Умножая справа на $\kappa v_{m-2} /(1+\kappa)$, имеем

$$
\mathcal{A}_{m-1} v_{m-2}-v_{m-2} \mathcal{A}_{m-2}=\tilde{\kappa}\left(\widetilde{\mathcal{A}}_{m-1} v_{m-2}-v_{m-2} \mathcal{A}_{m-2}\right),
$$

где

$$
\widetilde{\mathcal{A}}_{m-1} \equiv v_{m-1}^{\dagger} \widetilde{\mathcal{A}}_{m} v_{m-1}, \quad \tilde{\kappa}=\frac{\kappa}{1+\kappa} .
$$

Правая часть этого равенства по форме напоминает левую, но с заменой $\mathcal{A}_{m-1}$ на $\widetilde{\mathcal{A}}_{m-1}$, т. е. на величину, значение которой нам известно в явном виде. Подставляя данное выражение в уравнение (53) с $k=m-2$, получаем

$$
-v_{m-2}^{\dagger}\left(v_{m-2} \mathcal{A}_{m-2}-\widetilde{\mathcal{A}}_{m-1} v_{m-2}\right)+\frac{1}{\tilde{\kappa}}\left(v_{m-3} \mathcal{A}_{m-3}-\mathcal{A}_{m-2} v_{m-3}\right) v_{m-3}^{\dagger}=0 .
$$

Форма данного уравнения идентична (55), но в ней проведены замены

$$
m \rightarrow m-1, \quad \widetilde{\mathcal{A}}_{m} \rightarrow \widetilde{\mathcal{A}}_{m-1}=v_{m-1}^{\dagger} \widetilde{\mathcal{A}}_{m} v_{m-1}, \quad \kappa \rightarrow \tilde{\kappa}=\frac{\kappa}{1+\kappa} .
$$

Тем самым мы описали один шаг индукции, а именно $m \rightarrow m-1$.

Так как сама схема индукции установлена, перейдем к анализу того, какие условия налагает исходное уравнение $(55)$ на поле $\mathcal{A}_{m-1}$ в пределе $\kappa \rightarrow 0$. Во-первых, в этом пределе мы имеем

$$
\left(v_{m-2} \mathcal{A}_{m-2}-\mathcal{A}_{m-1} v_{m-2}\right) v_{m-2}^{\dagger}=0 .
$$

Умножая на $v_{m-2}$ справа и на $v_{m-2}^{\dagger}$ слева, получаем

$$
\mathcal{A}_{m-2}=v_{m-2}^{\dagger} \mathcal{A}_{m-1} v_{m-2} \text {. }
$$

Это означает, что $\mathcal{A}_{m-2}$ - ограничение поля $\mathcal{A}_{m-1}$ на подпространство $V_{m-2}$ (вспомним вид (49) матрицы $\left.v_{m-2}\right)$. Подставляя $(59)$ в $(58)$, получаем дополнительное условие:

$$
\left(1-v_{m-2} v_{m-2}^{\dagger}\right) \mathcal{A}_{m-1} v_{m-2} v_{m-2}^{\dagger}=0 .
$$

Это условие означает, что матрица $\mathcal{A}_{m-1}$ является блочной нижнетреугольной (если мы считаем, что нижний правый блок соответствует подпространству $V_{m-2} \subset V_{1}$, а верхний левый - подпространству $\left.V_{m-2}^{\perp} \subset V_{1}\right)$. Уравнение (55) содержит в себе, однако, больше информации, чем то, что мы получили, перейдя напрямую к пределу $\kappa \rightarrow 0$ в самом уравнении. Действительно, после умножения справа данного 


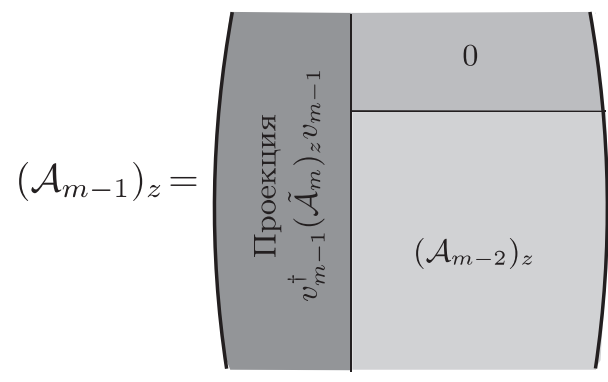

a

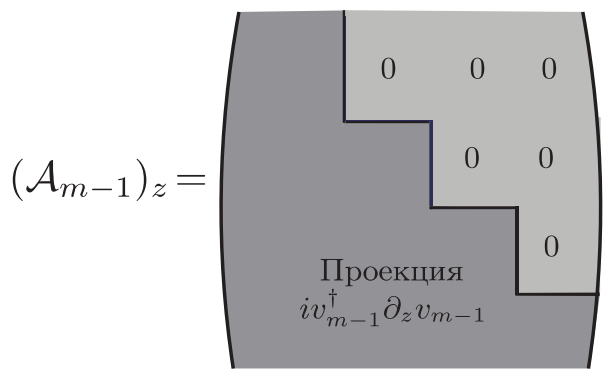

б

Рис. 4. Первый шаг рекуррентной процедуры, определяющий данную форму матрицы (а), и итоговая форма матрицы $\left(\mathcal{A}_{m-1}\right)_{z}(б)$.

уравнения на $1-v_{m-2} v_{m-2}^{\dagger}$ параметр $\kappa$ полностью пропадает из уравнения, и остается условие

$$
\left(\mathcal{A}_{m-1}-v_{m-1}^{\dagger} \widetilde{\mathcal{A}}_{m} v_{m-1}\right)\left(1-v_{m-2} v_{m-2}^{\dagger}\right)=0 .
$$

Это можно интерпретировать таким образом: “левый столбец" матрицы $\mathcal{A}_{m-1}$ полностью определяется значением $\widetilde{\mathcal{A}}_{m}$. В конечном счете уравнения (59)-(61) приводят к тому, что матрица $\mathcal{A}_{m-1}$ имеет вид, изображенный на рис. $4 \mathrm{a}$.

Повторяя только что описанную процедуру, в итоге мы приходим к форме матрицы $\left(\mathcal{A}_{m-1}\right)_{z}$, изображенной на рис. 4 .

Подставим теперь полученные калибровочные поля в лагранжиан (51). Как и ранее в п. 2.2 , легко показать, что ковариантные производные $\mathscr{D}_{z} v_{k}($ при $k<m-1$ ) имеют порядок $\kappa$ и обращаются в нуль в пределе $\kappa \rightarrow 0$. Действительно, умножая справа (52) на $v_{k-1}$, получаем

$$
\mathscr{D}_{z} v_{k-1}=v_{k}^{\dagger} \mathscr{D}_{z} v_{k} v_{k-1}, \quad k<m-1 .
$$

Умножая справа $(54)$ на $v_{m-2}$, аналогичным образом получаем

$$
\mathscr{D}_{z} v_{m-2}=\kappa v_{m-1}^{\dagger} \mathscr{D}_{z} v_{m-1} v_{m-2} \text {. }
$$

Из последнего равенства следует, что $\mathscr{D}_{z} v_{m-2}=O(\kappa)$ в пределе $\kappa \rightarrow 0$. Тогда, используя соотношение (62), можно рекуррентно доказать, что $\mathscr{D}_{z} v_{k}=O(\kappa)$ для всех $k<m-1$. Поэтому при подстановке данных выражений в лагранжиан (51) члены, содержащие эти ковариантные производные, обращаются в нуль в пределе $\kappa \rightarrow 0$. Тем самым остается вычислить

$$
\mathscr{D}_{z} v_{m-1}=\partial_{z} v_{m-1}+i v_{m-1}\left(\mathcal{A}_{m-1}\right)_{z}
$$

Напомним, что матрица $v_{m-1}$ содержит всю информацию о векторах в $\mathbb{C}^{N}$, параметризующих подпространства $V_{1} \subset \cdots \subset V_{m-1}$. Из условия нормировки (48) следует, что эти векторы ортонормированы. Разобьем их на $m-1$ наборов $\tau_{1}, \ldots, \tau_{m-1}$ в соответствии со следующим естественным принципом: векторы из $\tau_{1}$ лежат в пространстве $V_{1}$, векторы из $\tau_{2}$ лежат в пространстве $V_{2}$ и ортогональны векторам из $\tau_{1}$ и т. д. Тогда матрицу $v_{m-1}$ можно записать так: $v_{m-1}=\left\{\tau_{1}, \tau_{2}, \ldots, \tau_{m-1}\right\}$. Введем 
также еще один набор $\tau_{m}$ ортонормированных векторов, лежащих в ортогональном дополнении к $V_{m-1}$. В таком случае условие нормировки (48) эквивалентно следующим условиям:

$$
\tau_{a}^{\dagger} \tau_{b}=\delta_{a b}, \quad a, b=1, \ldots, m .
$$

Можно также записать соотношение полноты

$$
\sum_{a=1}^{m} \tau_{a} \tau_{a}^{\dagger}=\mathbb{1}_{N}
$$

В этих обозначениях компоненты калибровочного поля $\left(\mathcal{A}_{m-1}\right)_{z}$ имеют вид (см. рис. 4б)

$$
\left[\left(\mathcal{A}_{m-1}\right)_{z}\right]_{a b}=\left\{\begin{array}{lll}
i \tau_{a}^{\dagger} \partial_{z} \tau_{b} & \text { при } & a \leqslant b, \\
0 & \text { при } \quad a>b,
\end{array} \quad a, b=1, \ldots, m-1 .\right.
$$

При подстановке в (64) получаем

$$
\mathscr{D}_{z} \tau_{a}=\partial_{z} \tau_{a}-\sum_{b=1}^{a} \tau_{b} \tau_{b}^{\dagger} \partial_{z} \tau_{a}
$$

и, используя (65), имеем

$$
\mathscr{D}_{z} \tau_{a}=\sum_{b=a+1}^{m} \tau_{b} \tau_{b}^{\dagger} \partial_{z} \tau_{a}, \quad a=1, \ldots, m-1 .
$$

Подставляя данное выражение в формулу (51), получаем окончательное выражение для лагранжиана:

$$
\mathcal{L}=\sum_{a=1}^{m-1} \sum_{b=a+1}^{m} \operatorname{Tr}\left(\left(J_{b a}\right)_{z}^{\dagger}\left(J_{b a}\right)_{z}\right), \quad\left(J_{b a}\right)_{z}=\tau_{b}^{\dagger} \partial_{z} \tau_{a}
$$

Данный лагранжиан совпадает со введенным в работе [1] в случае, когда таргет-пространство есть пространство флагов (4), а комплексная структура выбрана таким образом, чтобы блочные (верхне- или нижне-) треугольные матрицы отвечали голоморфному подпространству $\mathfrak{m}_{+}$. Данное требование соответствует ограничению $b>a$ в сумме, входящей в лагранжиан (66).

\section{4. ЗАКЛЮЧЕНИЕ И ДАЛЬНЕЙШИЕ ПЕРСПЕКТИВЫ}

В представленной статье был продолжен анализ двумерных $\sigma$-моделей, введенных в работе [1]. Таргет-пространства этих моделей представляют собой комплексные однородные многообразия, а входящее в лагранжиан $B$-поле специального вида обеспечивает, что нётеров ток является плоским, и это позволяет построить представление нулевой кривизны для уравнений движения по классической формуле Полмайера (см. (3)). Весьма заметный класс пространств, попадающих в эту категорию, - это пространства флагов. В частном случае грассмановых многообразий они яляются симметрическими пространствами, при этом $B$-поле становится топологическим, а модели классически эквивалентны стандартным интегрируемым 
моделям с симметрическими таргет-пространствами, которые изучались, например, еще в работах [6], [7]. В остальных случаях, однако, модели являются новыми.

Известно, что самая естественная теория возмущений для $\mathbb{C P}^{N-1}$-модели состоит в изучении предела $N \rightarrow \infty$ и построении $1 / N$-разложения для различных наблюдаемых. Удобнее всего это сделать, пользуясь квазилинейной формулировкой, когда вводятся $N$ линейных "полей материи", удовлетворяющих квадратичным условиям (выполнение которых можно обеспечить при помощи введения в действие множителей Лагранжа) и взаимодействующих со вспомогательным $U(1)$-калибровочным полем. Данный подход известен начиная с работ [9], [10], и в его рамках были получены важные результаты в теории $\mathbb{C P}^{N-1}$-модели, например анализ сокращения аномалии в нелокальном сохраняющемся заряде после введения фермионов [12].

В настоящей статье построена квазилинейная формулировка для $\sigma$-моделей пространств флагов, введенных в работе [1]. Точно так же, как в $\mathbb{C P}^{N-1}$-случае введение вспомогательного $U(1)$-калибровочного поля можно связать с кэлеровым фактором плоского пространства $\mathbb{C}^{N}$, существует аналогичная конструкция кэлерова фактора для пространств флагов, представляющая собой естественный пример общей теории "колчанных представлений" [11] (данные для фактора задаются колчанной диаграммой). Отличие нашего случая состоит в том, что в лагранжиан (1) входит метрика Киллинга, вообще говоря, не являющаяся кэлеровой. В связи с этим процедура кэлерова фактора требует определенной модификации, при этом неизменными остаются калибровочная группа, по которой производится факторизация, и ее представления, по которым преобразуются так называемые "поля материи" модели. Мы показали явно, что после исключения вспомогательных калибровочных полей при помощи (алгебраических) уравнений движения получается стандартная (нелинейная) форма модели (1). Мы считаем, что этот результат послужит хорошей отправной точкой для анализа квантовых свойств модели.

ПРИЛОЖЕНИЕ А Исключение калибровочных полей из лагранжиана из п. 2.2

Запишем уравнения, которые получаются при вариации действия, отвечающего лагранжиану (33), по полям $\mathcal{A}_{\bar{z}}$ и $\mathcal{B}_{\bar{z}}$ :

$$
i \bar{u} \circ \mathscr{D}_{z} u=0 \quad \Longrightarrow \quad i \bar{u} \circ \partial_{z} u+\mathcal{A}_{z}+\bar{u} \mathcal{B}_{z} u=0
$$

и

$$
\mathscr{D}_{z} u \otimes \bar{u}+\kappa \sum_{k=1}^{3} \mathscr{D}_{z} v^{k} \otimes \bar{v}^{k}=0
$$

следовательно,

$$
\partial_{z} u \otimes \bar{u}+\kappa \sum_{k=1}^{3} \partial_{z} v^{k} \otimes \bar{v}^{k}-i \mathcal{A}_{z} u \otimes \bar{u}-i \mathcal{B}_{z}\left(u \otimes \bar{u}+\kappa \mathbb{1}_{2}\right)=0
$$

(мы использовали условия нормировки для упрощения выражений). При $\kappa \neq 0,-1$ можно воспользоваться формулой обращения

$$
\left(\kappa \mathbb{1}_{2}+u \otimes \bar{u}\right)^{-1}=\frac{1}{\kappa}\left(\mathbb{1}_{2}-\frac{1}{1+\kappa} u \otimes \bar{u}\right),
$$


чтобы выразить из предыдущей формулы $\mathcal{B}_{z}$ через $\mathcal{A}_{z}$ и поля материи:

$$
\mathcal{B}_{z}=-\frac{i}{1+\kappa}\left(\partial_{z} u-i \mathcal{A}_{z} u\right) \otimes \bar{u}-i \sum_{k=1}^{3} \partial_{z} v^{k} \otimes \bar{v}^{k} \circ\left(\mathbb{1}_{2}-\frac{1}{1+\kappa} u \otimes \bar{u}\right) .
$$

Подстановка в (П.1) выражения

$$
\bar{u} \mathcal{B}_{z} u=-\frac{i}{1+\kappa} \bar{u} \circ\left(\partial_{z} u-i \mathcal{A}_{z} u\right)-i \frac{\kappa}{1+\kappa} \sum_{k=1}^{3}\left(\bar{u} \circ \partial_{z} v^{k}\right) \cdot\left(\bar{v}^{k} \circ u\right)
$$

дает

$$
\frac{i \kappa}{1+\kappa}\left(\bar{u} \circ\left(\partial_{z} u-i \mathcal{A}_{z} u\right)-\sum_{k=1}^{3}\left(\bar{u} \circ \partial_{z} v^{k}\right) \cdot\left(\bar{v}^{k} \circ u\right)\right)=0 .
$$

Следовательно, мы получаем выражение для $\mathcal{A}_{z}$ :

$$
\mathcal{A}_{z}=-i \bar{u} \circ \partial_{z} u+i \sum_{k=1}^{3}\left(\bar{u} \circ \partial_{z} v^{k}\right) \cdot\left(\bar{v}^{k} \circ u\right) .
$$

Окончательный ответ для поля $\mathcal{B}_{z}$ получается при подстановке данного выражения в (П.4). Заметим, что этот вывод имеет смысл лишь при $\kappa \neq 0$, так как в противном случае выражение (П.5) тождественно обращается в нуль. Напротив, результирующие выражения (П.4), (П.6) несингулярны в пределе $\kappa \rightarrow 0$.

Из соотношения (П.2) легко видеть, что

$$
\mathscr{D}_{z} u=\partial_{z} u-i \mathcal{A}_{z} u-i \mathcal{B}_{z} u=\kappa\left(i \mathcal{B}_{z} u+\sum_{m=1}^{3} \partial_{z} v^{k}\left(\bar{v}^{k} \circ u\right)\right) .
$$

Как только что обсуждалось, поле $\mathcal{B}_{z}$ несингулярно в пределе $\kappa \rightarrow 0$, вследствие чего в этом пределе $\mathscr{D}_{z} u=O(\kappa)$ и

$$
\lim _{\kappa \rightarrow 0} \frac{1}{\kappa} \overline{\mathscr{D}_{z} u} \circ \mathscr{D}_{z} u=0 .
$$

Следовательно, остается вычислить значение $\mathscr{D}_{z} v^{k}$, подставив $\mathcal{B}_{z}$ из (П.4) и перейдя к пределу $\kappa \rightarrow 0$.

Несложное преобразование формулы (П.6) дает (см. определение (34))

$$
\mathcal{A}_{z}=i \sum_{m=1}^{3} \bar{w}^{m} \partial_{z} w^{m}
$$

При вычислении $\mathscr{D}_{z} v^{k}$ полезно следующее равенство:

$$
\bar{v}^{m} \circ(1-u \otimes \bar{u}) \circ v^{n}=P^{n m}-w^{n} \bar{w}^{m}=q^{n} \bar{q}^{m} .
$$

Отсюда

$$
\begin{aligned}
\mathscr{D}_{z} v^{k} & =\partial_{z} v^{k}-i \mathcal{B}_{z} v^{k}= \\
& =\partial_{z} v^{k}-\sum_{m=1}^{3} \partial_{z} v^{m} \bar{q}^{m} q^{k}-\left(\partial_{z} u+\sum_{m=1}^{3} \bar{w}^{m} \partial_{z} w^{m} u\right) w^{k},
\end{aligned}
$$


и, используя (37), мы имеем

$$
\mathscr{D}_{z} v^{k}=\sum_{m=1}^{3} \partial_{z} v^{m}\left(\bar{w}^{m} w^{k}+\bar{s}^{m} s^{k}\right)-\left(\partial_{z} u+\sum_{m=1}^{3} \bar{w}^{m} \partial_{z} w^{m} u\right) w^{k} .
$$

Из определения (34) вектора $w^{k}$ следует, что

$$
\sum_{m=1}^{3} \partial_{z} v^{m} \bar{w}^{m}-\partial_{z} u=-\sum_{m=1}^{3} v^{m} \partial_{z} \bar{w}^{m}
$$

Кроме того, так как $\sum_{m=1}^{3} \bar{s}^{m} v^{m}=0$, мы получаем

$$
\sum_{m=1}^{3} \partial_{z} v^{m} \bar{s}^{m}=-\sum_{m=1}^{3} v^{m} \partial_{z} \bar{s}^{m}
$$

Поэтому предыдущую формулу можно упростить:

$$
\mathscr{D}_{z} v^{k}=-w^{k} \sum_{m=1}^{3}\left(v^{m} \partial_{z} \bar{w}^{m}+u \bar{w}^{m} \partial_{z} w^{m}\right)-s^{k} \sum_{m=1}^{3} v^{m} \partial_{z} \bar{s}^{m} .
$$

Теперь несложно вычислить лагранжиан (33) при подстановке в него вспомогательных калибровочных полей и в пределе $\kappa \rightarrow 0$. Получим следующий результат:

$$
\mathcal{L}=\left|\sum_{m=1}^{3} \bar{q}^{m} \partial_{\bar{z}} s^{m}\right|^{2}+\left|\sum_{m=1}^{3} \bar{w}^{m} \partial_{\bar{z}} s^{m}\right|^{2}+\left|\sum_{m=1}^{3} \bar{q}^{m} \partial_{\bar{z}} w^{m}\right|^{2} .
$$

Это лагранжиан, введенный ранее в нашей работе [2].

ПРИЛОЖКНИЕ Б

\section{Квазилинейная формулировка при $\kappa>0$}

Чтобы дополнительно убедиться в несингулярности предела $\kappa \rightarrow 0$, рассмотрим снова лагранжиан в квазилинейной формулировке (33) при $\kappa \neq 0$,

$$
\mathcal{L}=\frac{1}{\kappa} \overline{\mathscr{D}_{z} u} \circ \mathscr{D}_{z} u+\sum_{k=1}^{3} \overline{\mathscr{D}_{z} v^{(k)}} \circ \mathscr{D}_{z} v^{(k)},
$$

и повторим процедуру приложения А, исключая калибровочные поля и приводя лагранжиан к стандартному виду нелинейной $\sigma$-модели пространства флагов.

Выражение для $\mathcal{A}_{z}$ не зависит от $\kappa$, поэтому оно остается неизменным:

$$
\mathcal{A}_{z}=i \sum_{m=1}^{3} \bar{w}^{m} \partial_{z} w^{m}
$$

Из (П.4) легко вывести, что

$$
\mathscr{D}_{z} u=\frac{\kappa}{1+\kappa}\left(-i \mathcal{A}_{z} u+\sum_{m=1}^{3} v^{m} \partial_{z} \bar{w}^{m}\right) .
$$


Простые преобразования формулы (П.4) позволяют представить $\mathcal{B}_{z}$ в следующем виде:

$$
\mathcal{B}_{z}=\left.\mathcal{B}_{z}^{\kappa}\right|_{\kappa=0}+i \mathscr{D}_{z} u \otimes \bar{u}
$$

Тогда

$$
\mathscr{D}_{z} v^{k}=\left.\mathscr{D}_{z}^{\kappa}\right|_{\kappa=0} v^{k}+w^{k} \mathscr{D}_{z} u,
$$

и, используя (П.8), (П.11), (П.12), мы имеем

$$
\mathscr{D}_{z} v^{k}=-\frac{1}{1+\kappa} w^{k} \sum_{m=1}^{3}\left(v^{m} \partial_{z} \bar{w}^{m}+u \bar{w}^{m} \partial_{z} w^{m}\right)-s^{k} \sum_{m=1}^{3} v^{m} \partial_{z} \bar{s}^{m} .
$$

Та же процедура, что приводит от (П.8) к (П.9), заключающаяся в возведении в квадрат выражений (П.12) и (П.13), дает в данном случае лагранжиан

$$
\mathcal{L}_{\kappa}=\left|\sum_{m=1}^{3} \bar{q}^{m} \partial_{\bar{z}} s^{m}\right|^{2}+\left|\sum_{m=1}^{3} \bar{w}^{m} \partial_{\bar{z}} s^{m}\right|^{2}+\frac{1}{1+\kappa}\left|\sum_{m=1}^{3} \bar{q}^{m} \partial_{\bar{z}} w^{m}\right|^{2} .
$$

Таким образом, мы получили $\sigma$-модель пространства флагов, однако с несколько несимметричной (“сплющенной”) метрикой (по сравнению с (39)). Тем не менее $S U(3)$-симметрия здесь также сохранена. Из формулы (П.14) очевидно, что предел $\kappa \rightarrow 0$ абсолютно гладкий.

Отметим также, что предел $\kappa \rightarrow \infty$ дает $\sigma$-модель с таргет-пространством $\mathbb{C P}^{2}$. При этом $\mathbb{C P}^{2}$ параметризуется вектором $s \in \mathbb{C}^{3}$, и в этом пределе можно полностью избавиться от полей $q, w$ в лагранжиане (П.14):

$$
\lim _{\kappa \rightarrow \infty} \mathcal{L}_{\kappa}=\left|\sum_{m=1}^{3} \bar{q}^{m} \partial_{\bar{z}} s^{m}\right|^{2}+\left|\sum_{m=1}^{3} \bar{w}^{m} \partial_{\bar{z}} s^{m}\right|^{2}=\sum_{m, n=1}^{3} \partial_{z} \bar{s}^{n} \partial_{\bar{z}} s^{m}\left(q^{n} \bar{q}^{m}+w^{n} \bar{w}^{m}\right) .
$$

Используя соотношение полноты (37), мы имеем

$$
\lim _{\kappa \rightarrow \infty} \mathcal{L}_{\kappa}=\sum_{m, n=1}^{3} \partial_{z} \bar{s}^{n} \partial_{\bar{z}} s^{m}\left(\delta^{m n}-s^{n} \bar{s}^{m}\right)
$$

В последнем выражении мы узнаем метрику Фубини-Штуди на $\mathbb{C P}^{2}$.

Благодарности. Я хочу поблагодарить К. Зарембо и О. Лехтенфельда за ценные замечания и особенно П. Зинн-Жюстена за полезные обсуждения колчанных многообразий. Я благодарен А. А. Славнову и моим родителям за поддержку.

\section{Список литературы}

[1] D. Bykov, "Complex structures and zero-curvature equations for $\sigma$-models", Phys. Lett. B, 760 (2016), 341-344, arXiv: 1605.01093.

[2] D. Bykov, "Integrable properties of $\sigma$-models with non-symmetric target spaces", Nucl. Phys. B, 894 (2015), 254-267.

[3] D. Bykov, "Classical solutions of a flag manifold $\sigma$-model", Nucl. Phys. B, 902 (2016), 292-301. 
[4] K. Pohlmeyer, "Integrable Hamiltonian systems and interactions through quadratic constraints", Commun. Math. Phys., 46:3 (1976), 207-221.

[5] A. D'Adda, P. Di Vecchia, M. Lüscher, "Confinement and chiral symmetry breaking in $\mathrm{CP}^{n-1}$ models with quarks", Nucl. Phys. B, 152:1 (1979), 125-144.

[6] В. Е. Захаров, А. В. Михайлов, "Релятивистски-инвариантные двумерные модели теории поля, интегрируемые методом обратной задачи”, ЖЭЭТФ, 74:6 (1978), 1953-1973.

[7] H. Eichenherr, M. Forger, "On the dual symmetry of the non-linear sigma models", Nucl. Phys. B, 155:2 (1979), 381-393.

[8] D. Bykov, The Kähler metric of a blow-up, arXiv: 1307.2816.

[9] E. Cremmer, J. Scherk, "The supersymmetric nonlinear $\sigma$-model in four-dimensions and its coupling to supergravity", Phys. Lett. B, 74:4-5 (1978), 341-343.

[10] A. D'Adda, M. Lüscher, P. Di Vecchia, "A $1 / n$ expandable series of non-linear $\sigma$ models with instantons", Nucl. Phys. B, 146:1 (1978), 63-76.

[11] V. Ginzburg, "Lectures on Nakajima's quiver varieties", Geometric methods in representation theory. I (Grenoble, France, June 16 - July 4, 2008), Séminaires et Congrès, 24, pt. 1, ed. M. Brion, Soc. Math. France, Paris, 2012, 145-219.

[12] E. Abdalla, M. C. B. Abdalla, M. Gomes, "Anomaly cancellations in the supersymmetric $\mathrm{CP}^{(n-1)}$ model", Phys. Rev. D, 25:2 (1982), 452-460.

Поступила в редакцию 11.08.2017 